Fabrication of Beryllium Capsules with Copper-Doped Layers for NIF Targets: A Progress Report

M. McElfresh, J. Gunther, C. Alford, E. Fought, R. Cook, A. Nikroo, H. Xu, J. C. Cooley, R. D. Field, R. E. Hackenberg, A. Nobile

September 12, 2005

The Fusion Science and Technology 
This document was prepared as an account of work sponsored by an agency of the United States Government. Neither the United States Government nor the University of California nor any of their employees, makes any warranty, express or implied, or assumes any legal liability or responsibility for the accuracy, completeness, or usefulness of any information, apparatus, product, or process disclosed, or represents that its use would not infringe privately owned rights. Reference herein to any specific commercial product, process, or service by trade name, trademark, manufacturer, or otherwise, does not necessarily constitute or imply its endorsement, recommendation, or favoring by the United States Government or the University of California. The views and opinions of authors expressed herein do not necessarily state or reflect those of the United States Government or the University of California, and shall not be used for advertising or product endorsement purposes. 


\title{
Fabrication of beryllium capsules with copper-doped layers for NIF targets: A progress report
}

Michael McElfresh, Janelle Gunther, Craig Alford, Eric Fought, and, Robert Cook, NIF, Lawrence Livermore National Laboratory, Livermore, CA 94550.

Abbas Nikroo and Hongwei Xu, General Atomics, P.O. Box 85608, San Diego, CA 92186-5608.

Jason C. Cooley, Robert D. Field, Robert E. Hackenberg, and Art Nobile, Materials Division, Los Alamos National Laboratory, Los Alamos, NM,

\begin{abstract}
The sputtering of beryllium (Be) has been used at LLNL for nearly 30 years in the fabrication of laser targets. Several years ago the prospect of using sputtering to fabricate spherical Be capsules for National Ignition Facility (NIF) targets began to be explored and a basic strategy was developed that involved sputtering down onto plastic mandrels bouncing in a pan. While this appears to be very straightforward in principle, in practice sputtering has been used almost exclusively to make thin films ( $<1$ micron) on flat substrates. Thick films pose a significant challenge for sputtering while materials on spherical substrates are essentially unexplored. More recently, based on computational results, the point design for the first NIF ignition target capsule was specified as a Be capsule with $\mathrm{Cu}$-doped layers of specific thickness, each layer with a different concentration of copper. While the work described here was motivated by the need to make the layered capsules, the primary progress on Be capsules has been the development of a more complete metallurgical understanding of the materials that are fabricated and the beginning of the exploration of the relationship between the sputter processing and microstructure of these spherical samples. At least two barriers to growth to full thickness (i.e. 170 microns) have been identified and efforts to overcome these barriers are underway.
\end{abstract}

\section{INTRODUCTION}

The current capsule point design ${ }^{1}{ }^{2}$ for the first ignition experiments at the National Ignition Facility (NIF) ${ }^{3}$ in 2010 is a graded copper-doped beryllium capsule. The full details of this design can be found elsewhere, but in Fig. 1 we show the relevant capsule specification with regard to the dopant layer positions and concentrations. There are roughness specifications on both the internal and external surfaces, as well as the interfaces between layers. ${ }^{4}$ 


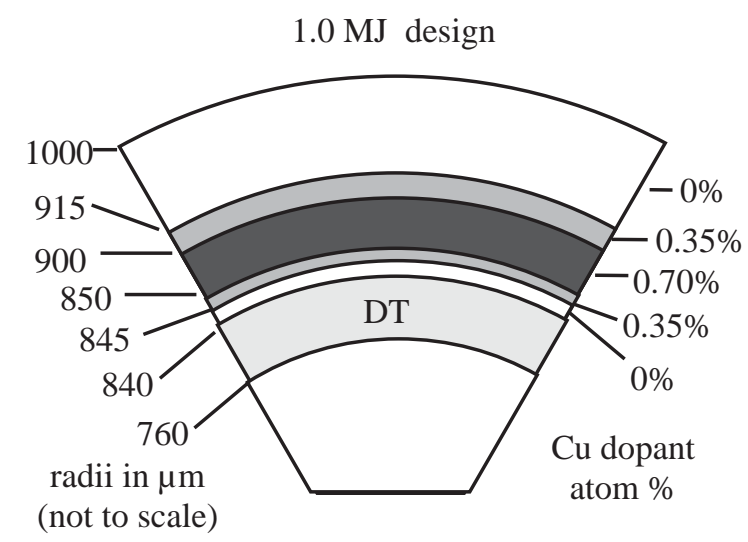

Figure 1. Basic capsule design for the current design 1 graded $\mathrm{Cu}$-doped Be capsule.

The fabrication of these capsules involves sputtering Be onto plastic mandrels ${ }^{5}$, the basic techniques have been described elsewhere. ${ }^{6}$ Sputtering is a well established method for depositing thin films onto flat surfaces, but its remains a significant challenge to coat a thick layer onto a spherical surface. We have now identified two barriers to sputter deposited growth to full thickness on spherical surfaces. In the case of the first of these barriers, there is a transition of the microstructure from a relatively dense columnar growth to a porous structure. The second barrier is a transition from columnar growth to a structure that has the appearance of particulate nucleated growth. The evidence so far shows that $\mathrm{Cu}$-doping has little effect on these microstructures and the transitions.

In addition to the layered structure, there is also a "density uniformity" specification, which for the work presented here can be expressed as a void density - void size and distribution specification. This is important since sputtered coatings are generally less than full density due to small voids that are formed in the growing coating. The current specification is a void fraction of less than $1 \%$ and that the volume of individual voids be less than $0.3 \mu \mathrm{m}^{3}$, though there is some flexibility because the uniformity specification can be met with a larger void fraction and smaller void volume. ${ }^{7}$ The actual spatial distribution of the voids may also be important if the distribution is not sufficiently random.

In what follows we will first briefly review our sputtering process and provide an overview of the characterization methods, then give a somewhat historical account of the process development and results from the past year. Since thick Be coatings on spherical substrates is a new area of coating science some of the coatings we have made and issues we have identified are of interest. We will conclude with our current plans.

\section{EXPERIMENTAL}

The $\mathrm{Cu}$-doped $\mathrm{Be}$ capsules were prepared by sputtering using separate $\mathrm{Cu}$ and Be sources onto 2 $\mathrm{mm}$ diameter glow deposition polymer (GDP) spherical mandrels. During sputtering the mandrels are bounced in a pan in order to uniformly distribute the coating over the surface. Pure Be was sputtered using four 2-inch MAC magnetron sputter guns arranged on a square, each gun pointing downward toward the center of the bounce pan. Sputter targets were fabricated from 
Brush Wellman grade S-65 (99.5\%). The Be sources are approximately 4 inches above the bounce pan. A 1.3-inch mini-MAC magnetron sputter gun centered between the four Be guns was used to sputter $\mathrm{Cu}$. An aperture was placed close to the source in order to reduce the $\mathrm{Cu}$ flux while allowing the an output level to remain high enough for reliable operation of the gun. Sputtering was typically done in a 3.7 mTorr Ar atmosphere with the Be sputter guns operating at 300 watts per gun. The $\mathrm{Cu}$ gun power was varied between 4 and 8 watts for different $\mathrm{Cu}-$ doping levels. The Ar gas was introduced into the sputter chamber via a flanged path in front of the camera port thereby helping to keep the window to the camera clean. The deposition system is pumped using a cryopump and has a base pressure of $2 \times 10^{-8}$ Torr. Deposition runs are typically started after the system has attained $8 \times 10^{-8}$ Torr. The Ar sputter gas is obtained as boil off from liquid Ar and scrubbed using Ti sublimator.

The bounce pan was driven using either a piezo-electric drive mechanism using an amplified Wavetek signal source or a mechanical drive (LabWorks industrial shaker model ET126). When the piezo drive was used, the bounce frequency was repeatedly cycled from 15 to $20 \mathrm{kHz}$ over 0.05 second intervals. The mechanical drive was operated at $75 \mathrm{~Hz}$. Various bounce pans have been used including a pan with eight dimples used to isolate capsules during an initial coating step and a screen pan that has been explored for eliminating debris accumulation in the pan.

The deposition system includes the ability to apply a voltage bias to the pan during deposition. It was found that an $-80 \mathrm{~V}$ bias tended to be the highest voltage that could be used without causing capsules to stick to each other and stop moving in the pan. As the coatings become thicker it is sometimes necessary to reduce the bias to keep capsules from sticking.

Be capsules are initially characterized for shell thickness, coating density, capsule roundness, smoothness, and basic microstructure. Coating thickness is measured by several techniques. Shells are radiographed after coating and the radiographic images analyzed using image analysis software to determine the wall thickness and to investigate wall thickness uniformity. The wall thickness measurements are corroborated by imaging fracture cross-sections of one of the capsules in a batch in the scanning electron microscope (SEM). The SEM fracture cross-section images are also used to make qualitative assessments of the microstructure.

Each shell is weighed to within $+/-3 \mu \mathrm{g}$ before and after coating. Each shell is measured for diameter and out of roundness using an optical microscope and the RMS surface finish is determined using interferometry. Those shells that have an RMS smoothness $<500 \mathrm{~nm}$ are analyzed with a "sphere-mapper," which is a modified atomic force microscope (AFM) used to make equatorial traces in order to produce roughness power spectra. Density is calculated using the mass of an individual shell, the average mandrel weight, the diameter, and thickness. Absolute-value density-measurement error-bars are currently about 10 percent primarily as a result of errors in measuring the wall thickness of the capsule, but efforts are underway to reduce these errors.

One capsule from each batch was also cross-sectioned and polished for additional analytical measurements. SEM imaging in the backscattered electron image (BEI) mode provides elemental contrast, i.e. Z-contrast, allowing the layers of different composition to be distinguished, helping define the sharpness of layer interfaces, and helping to identify the 
possible presence of other species like oxygen at the interfaces. On these same samples electron probe microanalysis (EPMA) uses wave dispersive spectroscopy (WDS) along with known standards in an SEM-like instrument with a specific geometry to provide quantitative compositional analysis (i.e. +/- 10 relative percent). Results are corrected for atomic number, absorption, and fluorescence effects (ZAF). Spatial resolution for analysis varies with Znumber, but is generally a few $\mu \mathrm{m}$ 's. The low $\mathrm{Z}$ of Be increases the sampling volume and therefore somewhat lowers the resolution obtainable. Using both the SEM and optical microscope, the polished cross sections also provide additional qualitative information on grain type, grain structure, and an idea about void density and distribution.

Specimens for transmission electron microscopy (TEM) were prepared using a focused ion beam (FIB). Using this technique, specific microstructural features can be removed from the polished surface of an optical microscopy specimen using a Ga ion beam. The specimen is then attached to a grid and finally thinned for TEM. Specimens were removed with their surfaces either parallel or perpendicular to radial direction. In the TEM, grain structure, void structure and distribution, second phase composition/crystal structure, and crystallography of microstructural features can be analyzed at high resolution.

Analysis of the crystallographic growth directions for the grains was performed using both TEM techniques and Electron Backscattered Diffraction (EBSD). The EBSD technique is performed in the SEM instrument and takes advantage of the de Broglie wave nature of the electron beam. Electron diffraction patterns are captured inside the SEM chamber on a fluorescent screen and recorded on a CCD camera. These patterns can be analyzed via computer algorithms to identify unknown phases by their crystal structure or determine the crystallographic orientations of a known phase. By taking such patterns from an array of points (or pixels), a data set can be generated in which orientation information is associated with each pixel. False color images can then be generated in which colors represent crystallographic orientations. Data from this technique, known as Orientation Imaging Microscopy (OIM), are usually presented as the crystallographic orientation parallel to the specimen plane normal; however, the full orientation information (e.g. rotation of the crystallographic orientation about this normal) is available in the data set. This allows detailed analyses to be performed, such as misorientations across boundaries and crystallographic directions/planes associated with specific features in the microstructure (e.g. the growth directions of the columnar grains in the current investigation).

\section{RESULTS AND DISCUSSION}

\section{A) First Thick Run (FTR)}

For our first attempt to make a full thickness capsule with doped-Cu layers it was decided that we would work to produce the specified $\mathrm{Cu}$-doping compositions of the layered capsule design but we would not work to produce the specified layer thicknesses. The details of the coating procedure utilized were based on the accumulated experience at the time. Calibrations of the $\mathrm{Cu}-$ doping level for the commonly used conditions (e.g. 300 watts per Be gun) were undertaken prior to starting the full thickness run. Approximate composition results from X-ray fluorescence (XRF) measurements on flat samples were used to set the $\mathrm{Cu}$ sputter gun power and aperture 
size. Some $2 \mathrm{~mm}$ GDP mandrels were coated with $\mathrm{Be}-\mathrm{Cu}$ at target values of 0.35 atomic percent and 0.7 percent. These capsules were sent out for neutron activation analysis in order to get an additional calibration of the composition on actual capsules. The values of $\mathrm{Cu}$-doping by neutron activation were found to be $0.34 \mathrm{~atm} \%$ and $0.65 \mathrm{~atm} \%$, while analysis with contact radiography gave values of $0.32 \mathrm{~atm} \%$ and $0.6 \mathrm{~atm} \% \mathrm{Cu}$. Measurements using wavelength dispersive x-ray analysis on an electron microprobe (EPMA) gave values of $0.33 \mathrm{~atm} \%$ and $0.64 \mathrm{~atm} \%$. All of these measurements had error bars on the order of 10 to 15 percent.

There was evidence in prior runs that plastic mandrels would deform if exposed to high sputtergun power levels and that they would stick together irreversibly early in the deposition process. To address these expectations mandrels were precoated in batches of eight capsules using a dimpled pre-coat pan with only two Be sputter guns operating at 50 watts each to grow a pure Be coating to about $0.1 \mu \mathrm{m}$ in thickness. Capsules from several precoat runs were then loaded into a smooth pan for the full coating run. Due to a history of capsules also sticking together during deposition the coating process was visually monitored by a person thereby limiting deposition runs to about 6.5 hours per day which, at a deposition rate of about 1.4 microns per hour, resulted in about 9 microns of coating per day. The system remained pumping overnight between runs. During a series of runs the capsules were coated with pure Be for two days to a thickness of about $18 \mu \mathrm{m}$, then at a target $\mathrm{Cu}$-doping value of $0.35 \mathrm{~atm} \%$ for two days for a layer thickness of $\sim 18 \mu \mathrm{m}$, then $0.7 \mathrm{~atm} \%$ for three days for a layer thickness of $\sim 27 \mu \mathrm{m}$, and then $0.35 \mathrm{~atm} \%$ for another three days for a layer thickness of $\sim 27 \mu \mathrm{m}$. The deposition chamber was opened and three capsules were removed after the layer of each composition was complete. The final layer was to be pure Be to $60 \mu \mathrm{m}$ thickness, however on the second day of pure Be deposition it was observed that the capsules were not bouncing. Debris had accumulated in the bounce pan and was sticking to the capsule surfaces and the capsules were becoming very rough (i.e. dull/diffusive scattering) in appearance. The bounce pan drive amplifier was modified in order to roughly double the drive power and the capsules were rerun, however, the capsules still did not bounce and the run was therefore terminated. The capsules were about 80 to $90 \mu \mathrm{m}$ thick at the time of termination.

Shown in Fig. 2 is an SEM fracture cross-section of one of the 5-layer graded Cu-doped Be capsule from the first thick run. The capsule is about $90 \mu \mathrm{m}$ thick. Evidence of columnar growth is seen through all but the last 5 to 10 microns on the outer surface and there are indications of intermediate layers. The magnified image also shows that the nodules formed in the outer layer are poorly bonded (see arrows). This outer layer exhibits a growth morphology that is frequently attributed to nucleation by particles. ${ }^{8}$ 


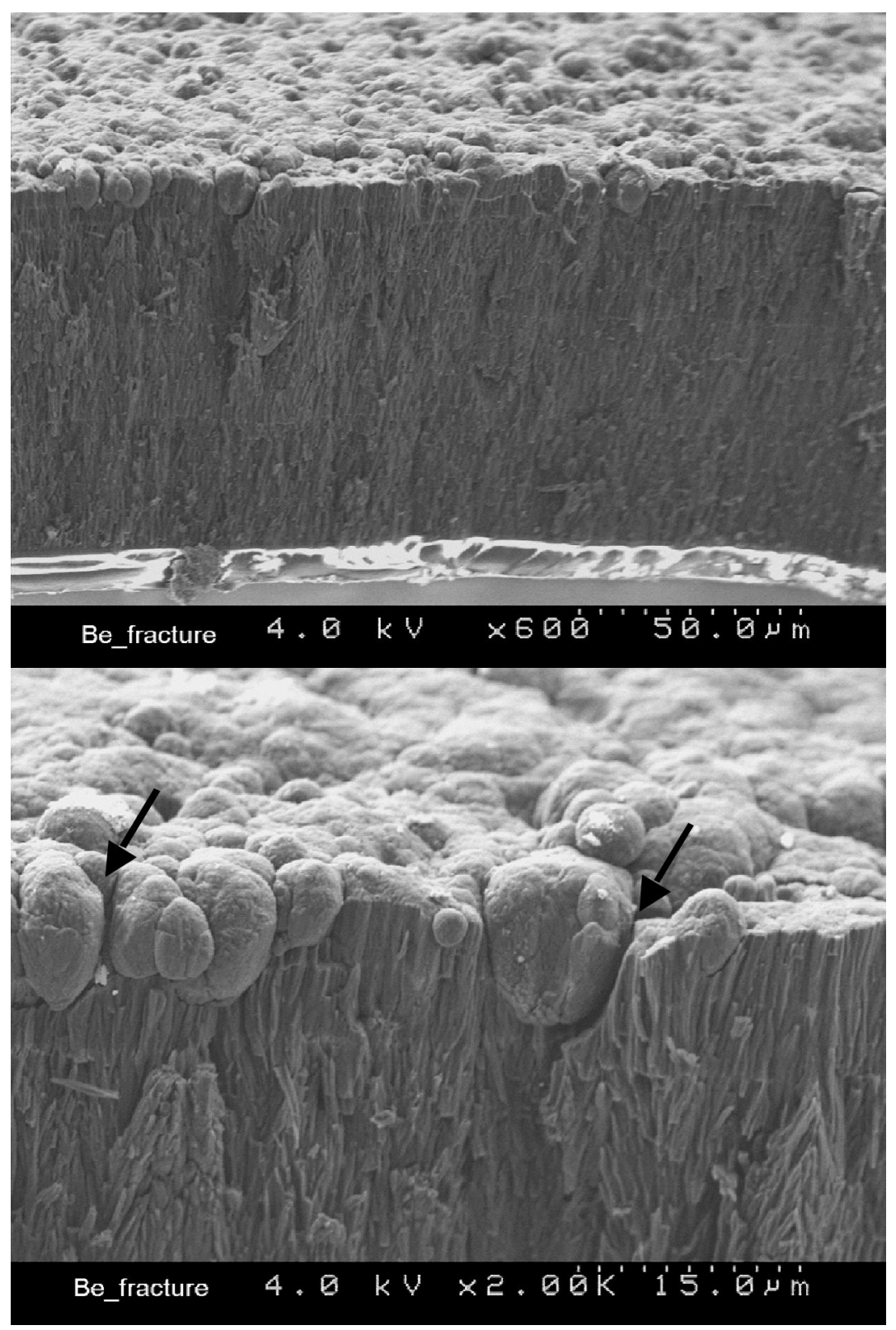

Figure 2. SEM images of a fracture cross-section of a 5-layer graded $\mathrm{Cu}$-doped Be capsule. Evidence of columnar growth is seen through all but the last 5-10 microns on the outer surface. Some indication of intermediate layers is also seen. The magnified image also shows that the nodules formed in the outer layer are poorly bonded (see arrows).

Shown in Fig. 3 is an image made of a polished section of the final $\mathrm{Cu}$-doped Be capsule wall when operating the SEM in the backscattered electron mode. The image shows the various layers with the brighter regions corresponding to higher concentrations of $\mathrm{Cu}$ and the darkest areas corresponding to pure Be layers. The light colored lines spaced at about $9 \mu \mathrm{m}$ we attribute to oxidation of the shells after the sputter guns are turned off at the end of day's run and this is supported by analysis with EDS showing increased oxygen content in those regions. The deposition chamber remained closed and pumping at vacuum overnight between the individual 
runs associated with a layer of a particular $\mathrm{Cu}$ doping level. At the completion of a layer of a given composition, three capsules removed for analysis and the system pumped down again.

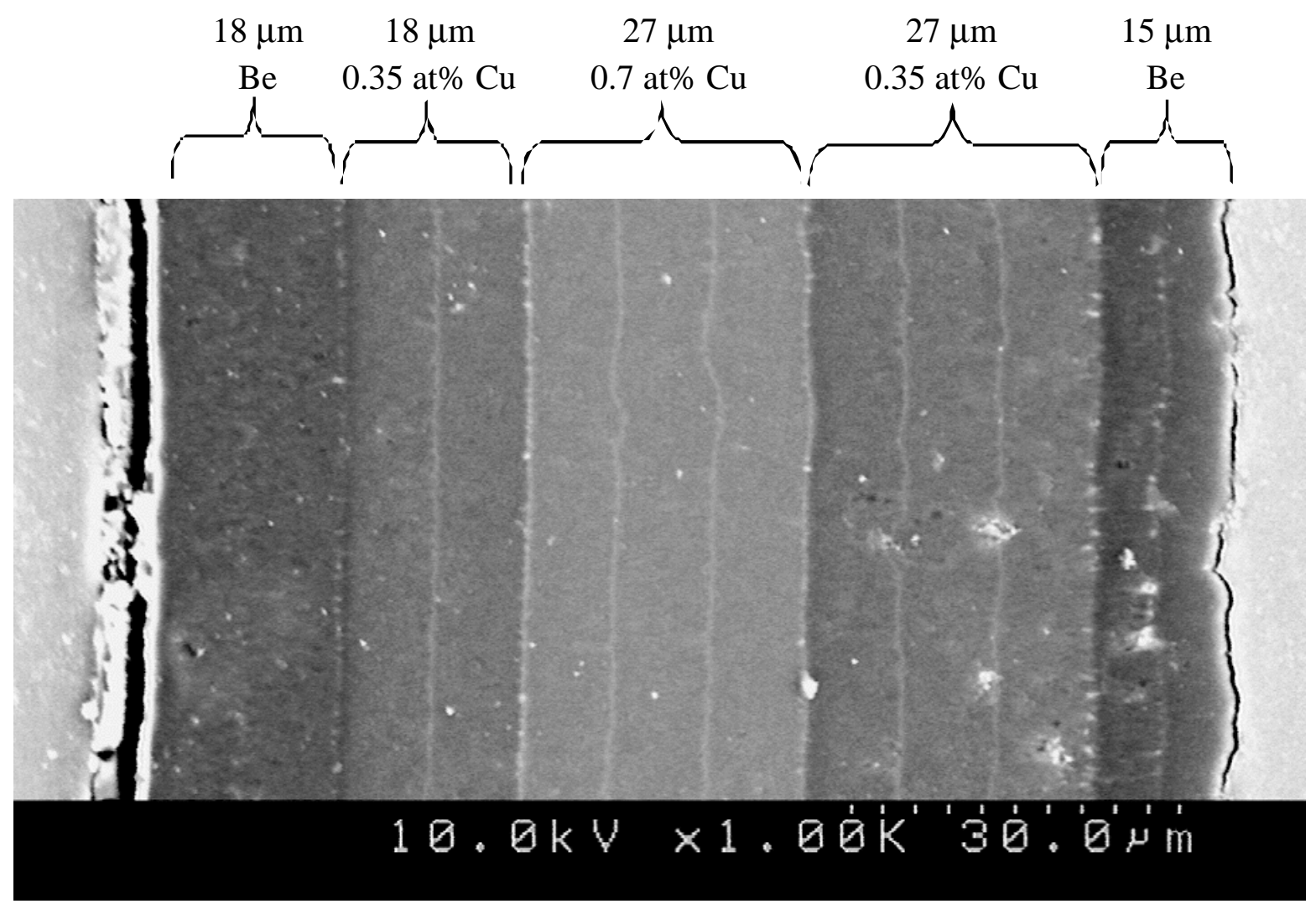

Figure 3. An SEM image of a polished cross-section of the capsule shown in Fig. 2. The change in brightness across the wall is indicative of changes in atomic number. The brighter areas correspond to regions where higher atomic number species are present. The brightest lines (nearly white) occur approximately every 8-9 $\mu \mathrm{m}$ and represent the interface between daily runs. Analysis with energy dispersive spectroscopy (EDS) of characteristic x-rays suggested increased oxygen content in these thin regions.

Transmission electron microscopy (TEM) was used to look more closely at microstructure, the grain growth, and voids. TEM samples were prepared using a FIB and shown in Fig. 4a is a sample cut parallel to the capsule radius exhibiting columnar structure radially directed with nearly full density grain boundaries. The voids that are evident at the grain boundaries (i.e. the lightest features) appear as small spherical or elongated bubbles found in diffuse strings with individual bubbles having typical dimensions of about $10 \mathrm{~nm}$ and extreme dimensions of about $200 \mathrm{~nm}$. A boundary layer that coincides with the stopping and starting of a run is visible. It appears that growth of grains terminates at the boundary layer and new grains start after the boundary layer. Figure $4 \mathrm{~b}$ shows a cut perpendicular to the capsule's radial direction. Small voids on the scale of a few nanometers are evident at grain boundaries. Their shape in this crosssection corroborates their bubble-like shape. Also evident is a fairly uniform grain size around one-half micron in cross-section. 
Note that while there is evidence of oxygen at the growth interruption there is no evidence of a crystalline $\mathrm{BeO}$ layer. We have looked for such a layer at higher resolutions and such a layer would have to be less than $1 \mathrm{~nm}$ thick to go undetected. The ring spacing in TEM Selected Area Diffraction (SAD) measurements of the radial specimen is consistent with the lattice constant of metallic beryllium with no evidence of second phase particles in any of the TEM micrographs and no evidence in the SAD of crystalline $\mathrm{BeO}$ or $\mathrm{Be}_{2} \mathrm{C}$.
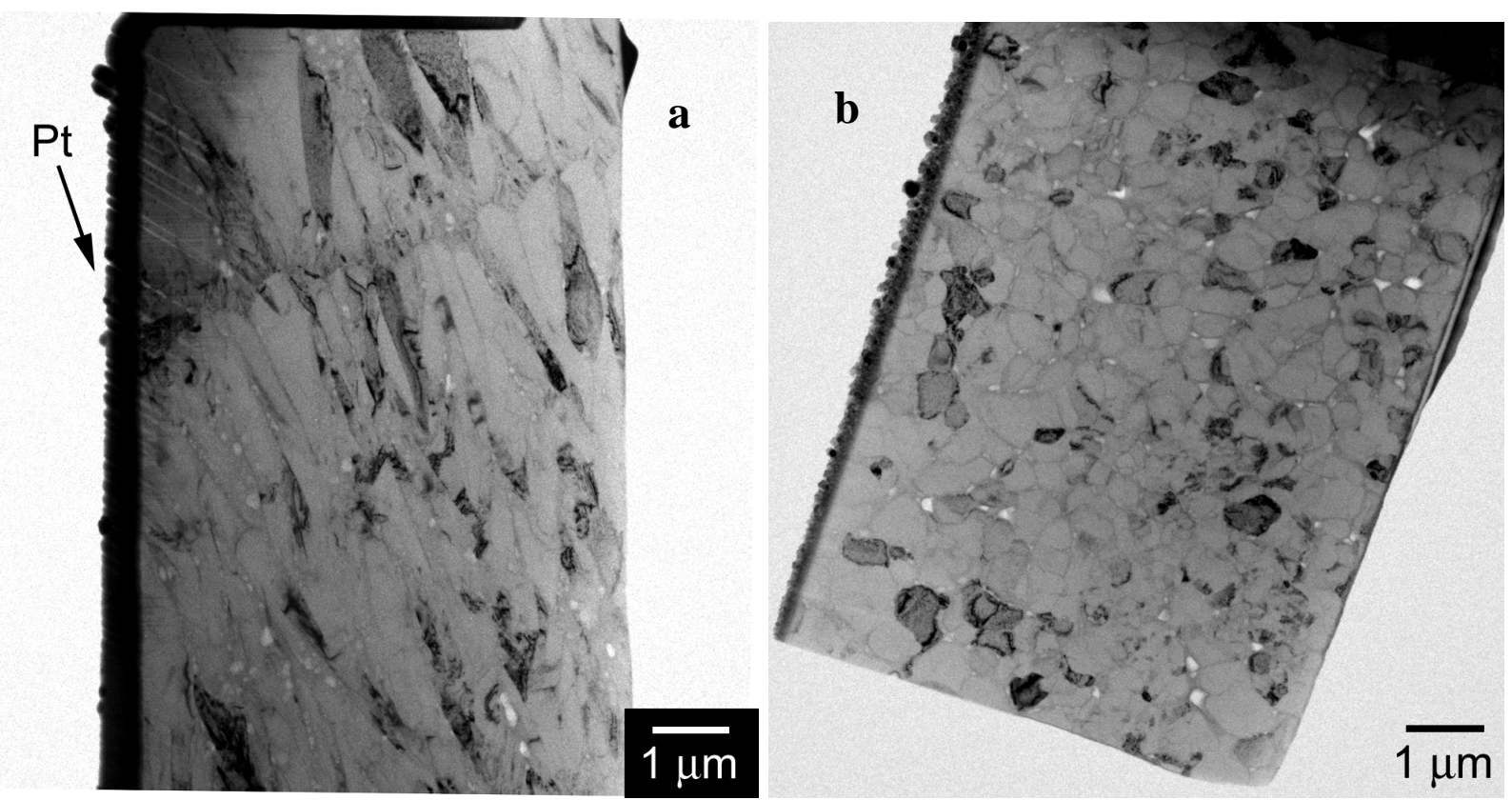

Figure 4. TEM micrographs show a radially directed columnar grain structure with breaks in the grain at boundaries formed presumably when the system was stopped overnight and when the chamber was opened. The voids are much smaller than seen in earlier capsules, forming chains of small "bubbles" rather than long pores lying parallel to the boundaries as seen previously. The TEM diffraction patterns taken from various areas in the grain boundaries showed no evidence of second phases.

A texture analysis of the samples was carried out using OIM. The OIM image in Fig. 5a is compiled from a series of diffraction measurements, which in combination with the associated key (i.e. Fig. 5b), shows the crystallographic orientation of individual crystallites normal to the sample surface. The long directions of the individual grains are oriented radially. Dark areas represent regions where crystallographic orientation cannot be determined because of the limited scattering (i.e. voids) or contributions to the patterns from more than one grain. Bands running perpendicular to the radial direction are presumably the boundaries produced between daily runs where grain orientation changes and are about $9 \mu \mathrm{m}$ apart consistent with the daily growth bands. The inverse pole figure in Fig. 5c shows that there is a (10-12) texture along the radial direction. The key for the inverse pole figure identifies the probability with respect to random with 1.00 representing random probability. This same texture had been observed using TEM techniques in an LLNL produced Be capsule made in 2002. ${ }^{9}$ The increased statistics associated with the OIM technique confirms that this is a general feature of the columnar growth. 

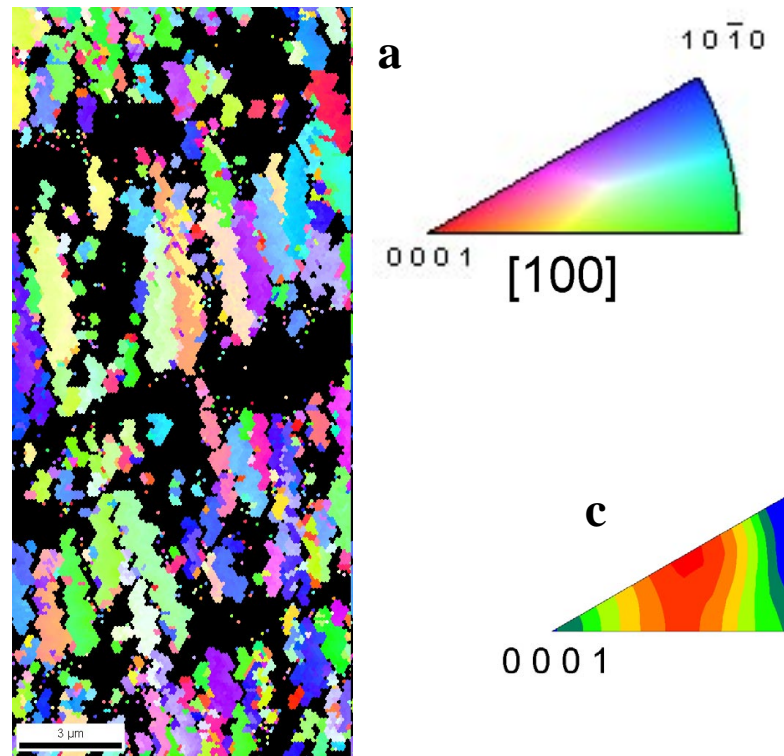

b

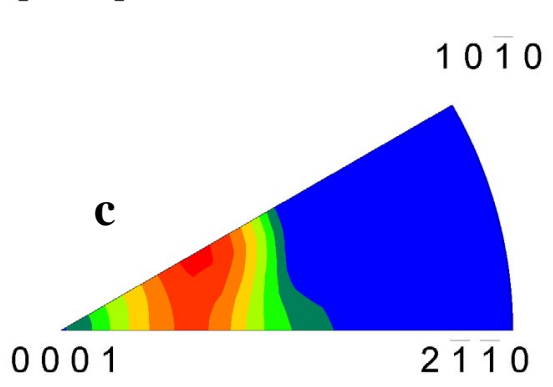

$\max =2.513$

2.400

2.015

1.691

1.419 d

1.191

1.000

0.839

$\min =0.230$

Figure 5. Orientational Imaging Microscopy (OIM) was used to determine the texture of the layered Be capsule. (a) The OIM image is compiled from a series of diffraction measurements and the key (b) shows the orientations normal to the surface. (c) The inverse pole figure along with the key (d) gives the probabilities of finding different orientations aligned along the radial direction. The highest probability is for the (10-12) direction.

The concerns about mandrel deformation due to rapid heating during the start of each sputtering session were also addressed in the procedure by ramping up the power of the Be sputter guns to full power (i.e. 300 watts per gun) over several tens of minutes (i.e. 30 to 60 minutes). However, since the $\mathrm{Cu}$ sputter source is always operated near its lowest power setting it was not ramped but rather was maintained at the final operating setting as the Be sources ramped up. The consequence of this procedure is that the $\mathrm{Cu}$ content will be too high while the Be sources are being ramped. Figure 6 shows composition profiles for $\mathrm{Cu}$ and $\mathrm{O}$ as a function of position measured by EPMA. The inner and outer walls of the capsule correspond to about 75 and 5 microns, respectively. In order to minimize interference from the mandrel and from the roughness of the outer layer of pure $\mathrm{Be}, 5-10 \mu \mathrm{m}$ on each side of the wall was not included in the analysis. The major steps in the $\mathrm{Cu}$ profile are evident, as are small peaks located at the boundaries between daily deposition runs. These smaller peaks are consistent with starting each daily run with the $\mathrm{Cu}$ gun at full power as the Be guns are ramped up in power. Also evident are high oxygen content at the boundaries both when the system is pumping overnight and when the system is opened to remove capsules between layers with different composition. 


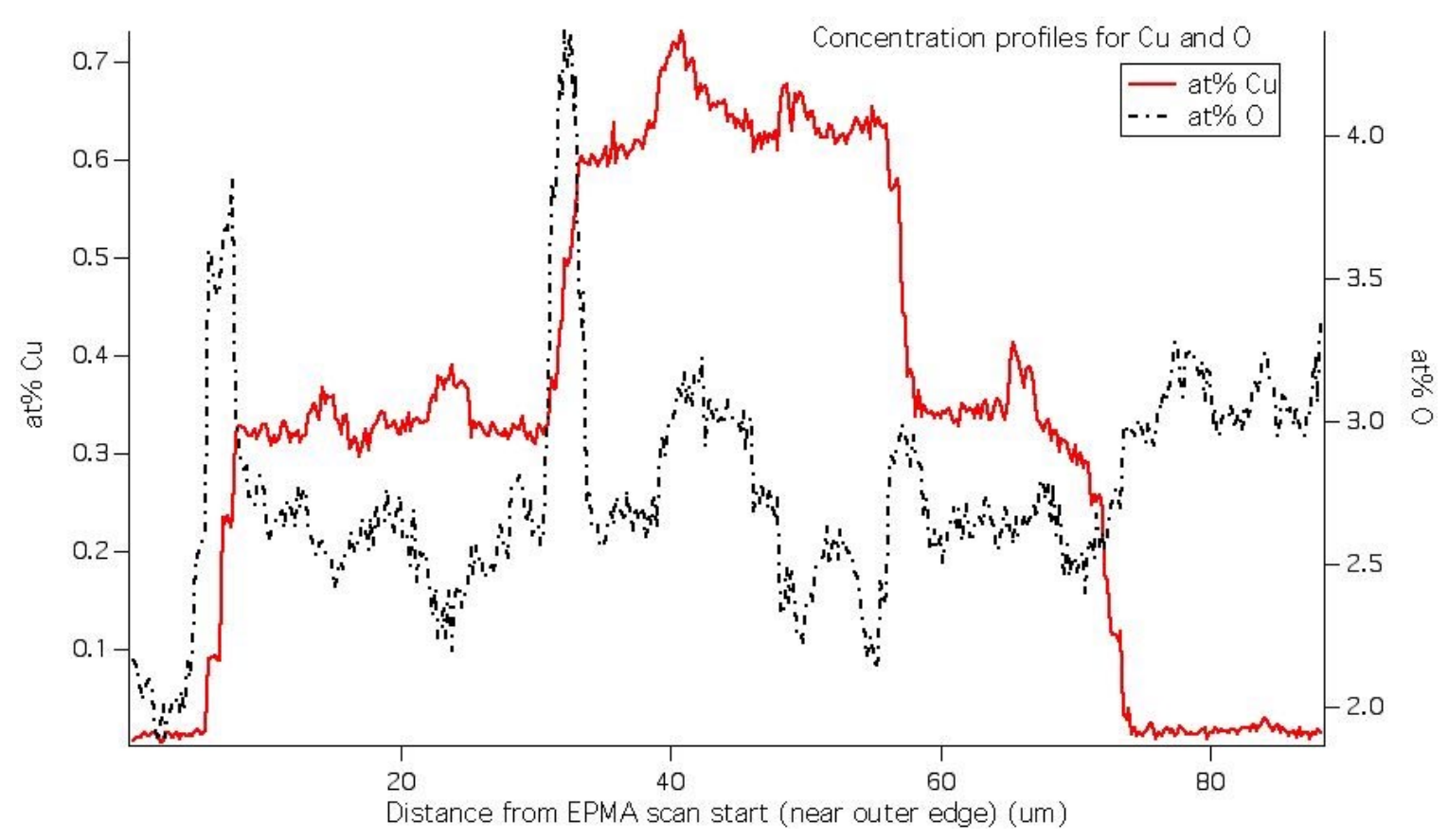

Figure 6. EPMA concentration profiles for $\mathrm{O}$ and $\mathrm{Cu}$. The steps between layers are shown clearly and occur at 0.33 and 0.65 at\% $\mathrm{Cu}$. Small peaks in the $\mathrm{Cu}$ content occur a significant percentage of the time in-between sputtering runs. Several large oxygen peaks are seen at the boundaries where the chamber was opened.

After the completion of each composition, each of which consisted of 2 or 3 individual daily runs, three capsules were removed for surface analysis with the sphere mapper. Figure 7 shows the power spectrum for the different stages of coating. The NIF design specification in Fig. 7 corresponds to the inner surface adjacent to the mandrel. Tolerances for the interfaces are reduced as one moves out from the inner wall. Because the thicknesses do not meet the design specifications it is not possible to directly compare these spectra to the design specifications, however a cursory comparison shows these results to be fair approximations to the specifications. 


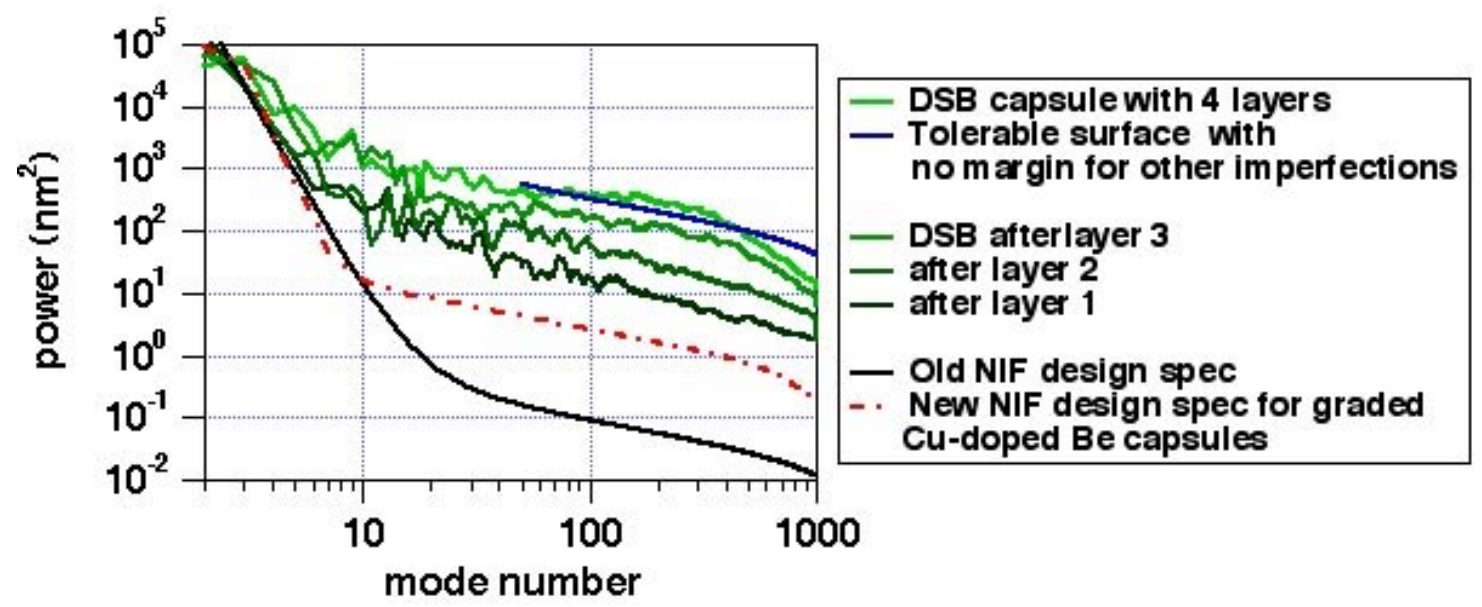

Figure 7. Shown are the successive sphere-mapping derived power spectra for the shells after each of the four layers of the FTR capsule was applied compared with calculated specifications.

\section{B) Second Thick Run (STR) Series}

Based on the analyses of the first thick run, numerous changes were made to the coater and coating process. A primary objective was to move to reliable operation that would allow, more efficient, 24 hours per day -7 days per week operation (i.e. 24/7) that would control the oxidation at the layer interfaces. In addition a series of tests were run to determine the need for low initial power exposures which was one of the reasons for the precoat step and the main reason for the ramp of the Be sputter guns. These tests did show that the precoat step and the power ramp could be eliminated. Other changes included replacing the piezo drive with an electro-mechanical drive mechanism, adding a shutter over the bounce pan, and adding an endHall type ion source for ion-assisted deposition.

A 24/7 run to produce a thicker capsule with the specified composition of $\mathrm{Cu}$, but now with the specified thickness values, was initially undertaken using ion-assisted deposition. Figure 8 shows an SEM fracture cross-section of a capsule produced in that run. Evident is a microstructural cross-over from what appears to be radial columnar growth near the mandrel to a non-radial structure deemed the "twisted" structure starting around 20 to 30 microns in thickness. Initially it was thought that this structure resulted in some way from the use of the ion-assisted deposition, however the same cross-over was subsequently observed under nearly identical deposition conditions but without the ion gun. 


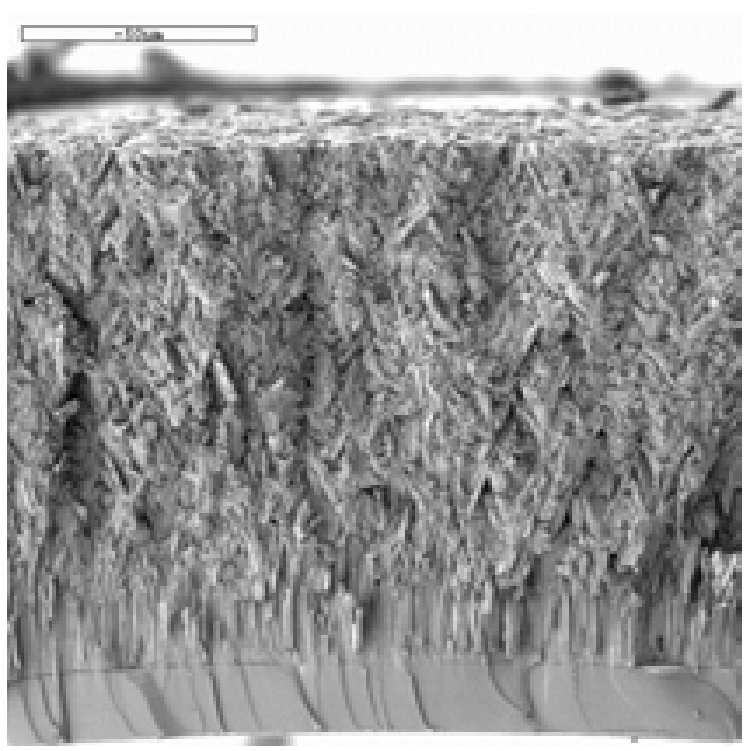

Figure 8. During continuous operation with 300 watt per gun deposition conditions, a crossover from columnar to the "twisted" structure appears at thickness values around 20 to 30 microns.

The capsules with material exhibiting the twisted microstructure appear to be fairly low in density (i.e. $\sim 1.6 \mathrm{~g} / \mathrm{cc}$ for a material that should have a density of $1.89 \mathrm{~g} / \mathrm{cc}$ if fully dense.) An interesting observation is that these capsules appeared very shiny (i.e. good specular reflectors) once the twisted growth mode is established and the final surface finishes were remarkably smooth, often less than $100 \mathrm{~nm}$ RMS. In most cases the bounce pan also was very smooth in the region of contact with the capsules. Figure 9 shows SEM micrographs of FIB cut samples. The faint lines from top to bottom in the images are artifacts caused by the gallium ion milling procedure. The pores are larger and longer than in the FTR capsule and are not generally oriented radially. Voids are now observed running along the length of grains and additional voids are observed at the junctions at the ends of crystallites. Pieces of the sample were observed to fall out during polishing making representative cross-sections very difficult to obtain. There were several observations associated with liquid uptake during polishing that suggested that the porosity created continuous paths through the twisted structure portion of the shell. ${ }^{10}$ 

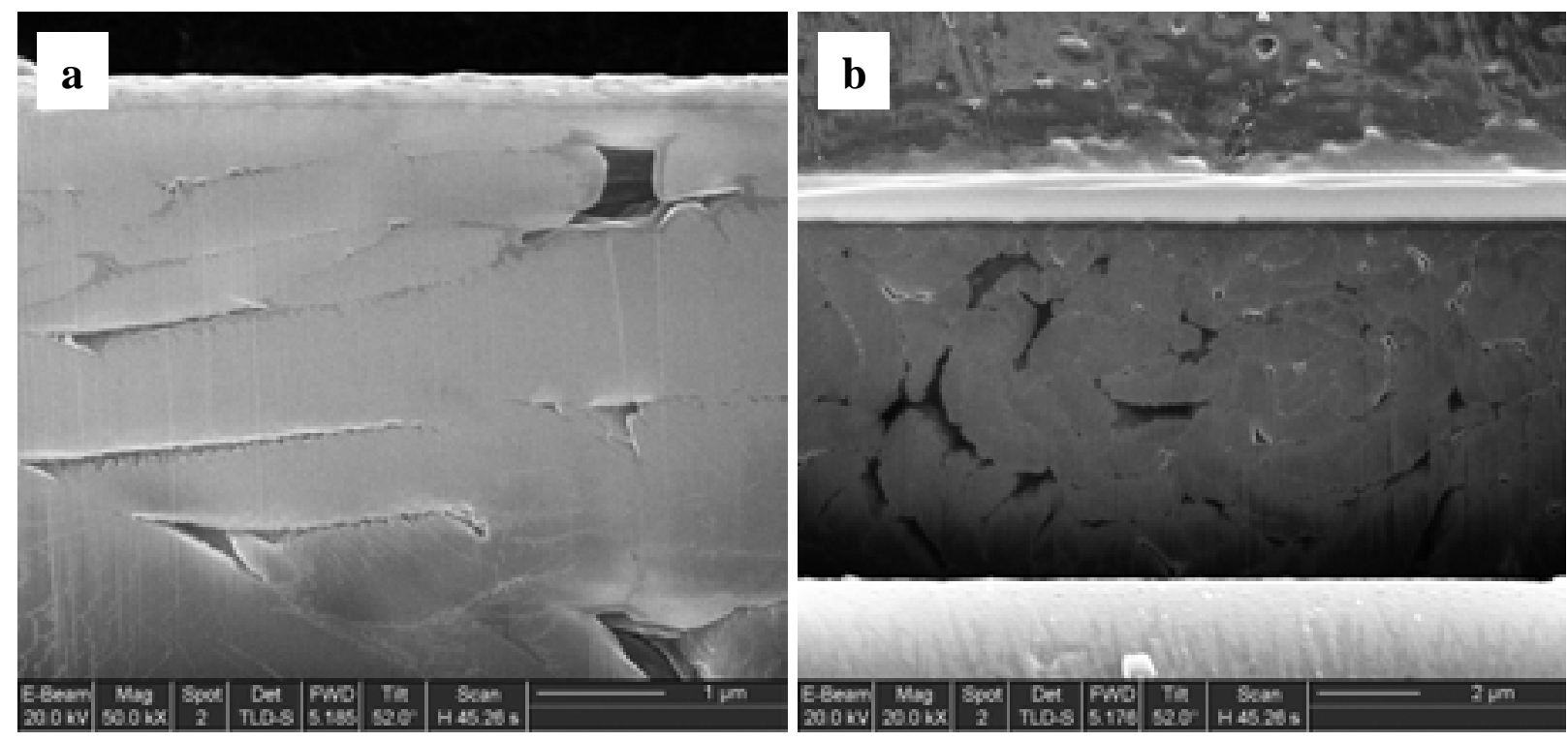

Figure 9. Here SEM micrographs, a) longitudinal cross-section, and b) transverse cross-section, of FIB-cut samples show the type of porosity for the "twisted" structure. There was evidence that the porosity creates continuous paths through the shell.

A search for secondary phases showed that little $\mathrm{BeO}$ or $\mathrm{BeC}$ was present in the void regions. SAD showed ring patterns consistent with beryllium and no evidence of crystalline second phases. Phases like $\mathrm{BeO}$ and $\mathrm{BeC}$ would manifest as faint rings in the SAD that could not be indexed to the lattice constant of beryllium.

In the SEM fracture cross section of the STR sample in Fig. 8 it is evident that the Be crystallites generally do not grow along the radial direction of the capsule. The OIM image for the twisted structure shows the material to differ significantly from the IOM data for the radial columnar texture of the FTR capsule. Figure 10 shows inverse pole figures for the c-axis aligned along both the capsule's radial direction and the local growth direction. Texture analysis from this OIM data on the twisted structure revealed a net c-axis texture along the radial direction for the capsule (Fig. 10b) however when the texture direction (i.e. RD) was chosen to lie along a local growth direction a (10-12) texture was observed that is closer to that previously seen for the FTR material (see Figs. 10a and Fig. 5c). 

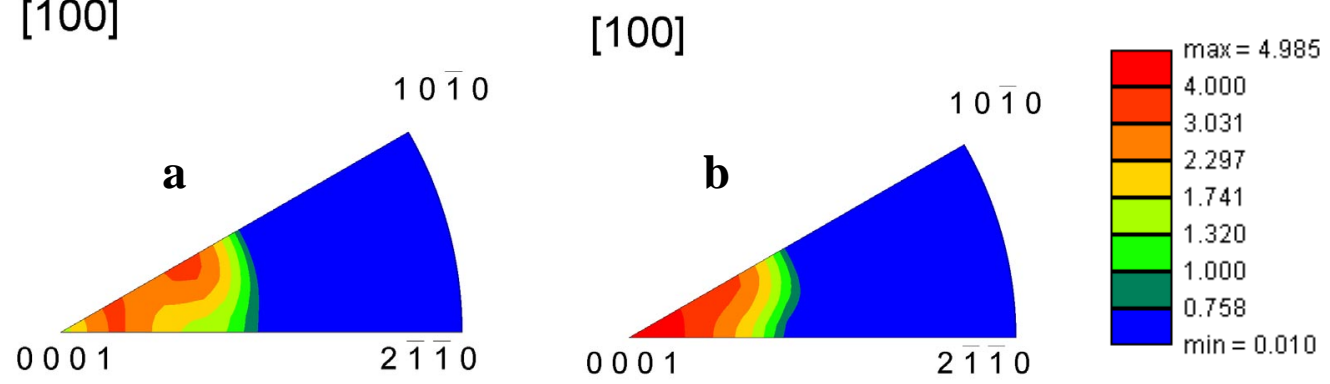

Figure 10. Inverse pole figures generated from the OIM data using (a) the local growth direction as the texture direction (RD or rolled direction) and (b) using the radial direction as RD.

A polished cross-section of the STR capsule is shown in Fig. 11 and was subsequently imaged with SEM and EPMA. The twisted structure is evident in the outer layers. The image was taken in backscatter mode, which provides the atomic number contrast seen in the image with the lighter shades corresponding to larger atomic number. Due to the continuous 24/7 operation of the coater bright bands (attributed to $\mathrm{O}$ ) are no longer seen at the interfaces. 


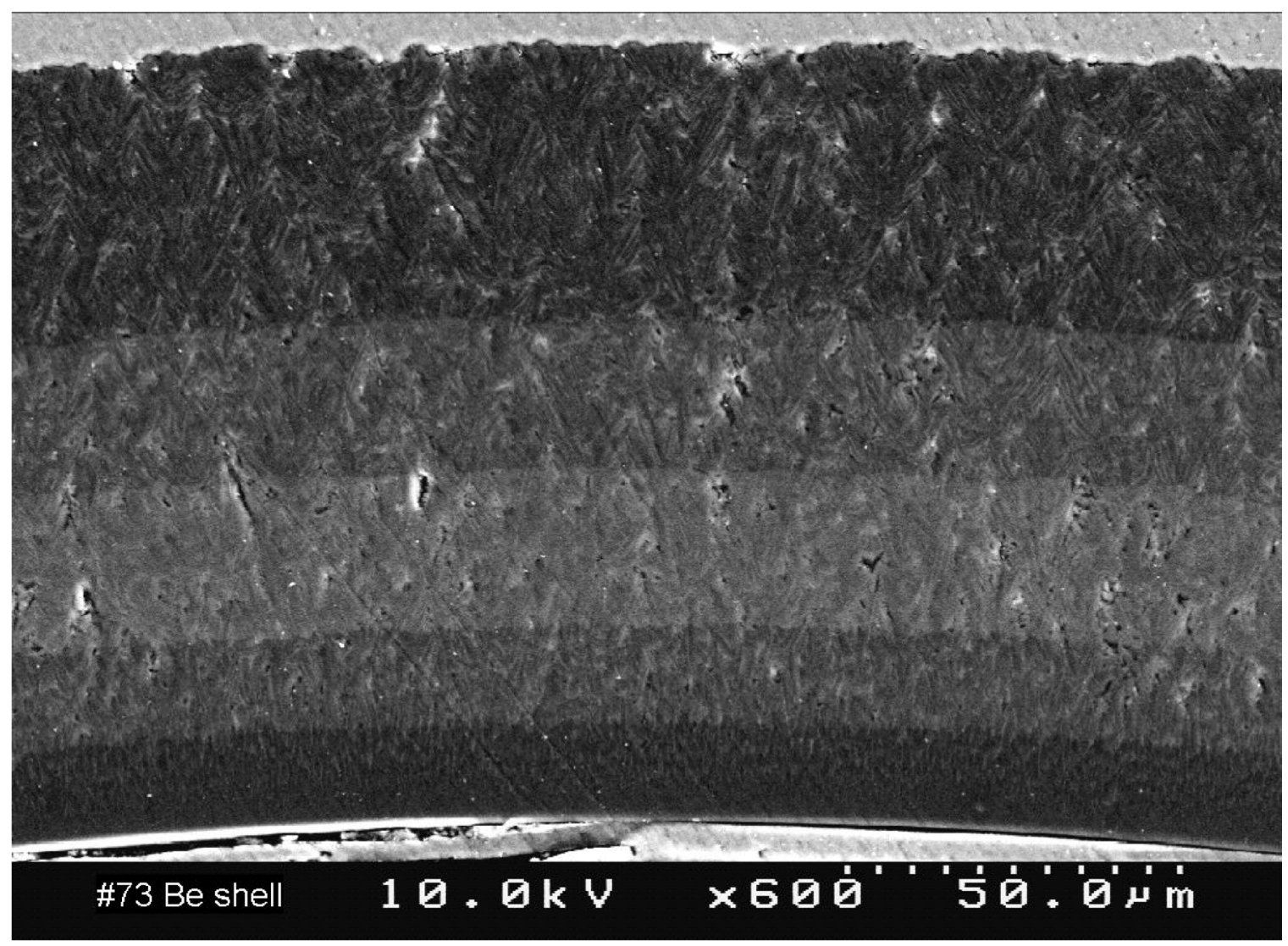

Figure 11. SEM image of the polished cross-section of a STR capsule taken in backscatter mode, which provides the atomic number contrast seen in the image. Due to the $24 / 7$ operation of the coater the bright bands seen in Fig. 3 (attributed to $\mathrm{O}$ ) are no longer seen at the interfaces.

\section{C) Recent results and directions}

The first thick capsule run (FTR) produced a product with characteristics that helped to guide development of the deposition process and modifications to the deposition system. The resulting twisted structure that was produced once the system was capable of 24/7 operation and uninterrupted deposition came as a surprise and required that a new process strategy be developed. Because of its low density, fairly large pores, and the possible continuously porous nature, this is a structure that we intend to suppress. The current strategy for making full thickness capsules is to work to suppress the twisted structure and once this is accomplished to work to optimize the microstructure of the retained structure with, for example, ion-assisted deposition to get the material to its maximum density. It appears that the columnar structure was inadvertently retained in the FTR capsules as a consequence of the boundary layers formed when the system sat pumping overnight and when the system was opened for capsule removal. However, when the Be shell reached 80 to 90 microns in thickness there was a breakdown from columnar growth to a structure that resembled particulate initiated growth (PIG).

"Cycling" a system parameter in order to affect microstructure has been used in the past as a general method for controlling microstructure. Some of the suggestions of parameters to cycle include Ar sputter gas pressure, voltage bias, temperature of the capsules, sputter gun power, 
contaminant gas concentration, deposition rate, and ion gun current. The first cycling method we have explored was to cycle the Ar sputter gas pressure. ${ }^{11}$ The pressure values at the ends of the cycle were selected so that the system is cycled between conditions that alternate between layers in tension and in compression. In addition the thickness values of layers are selected so that the total amount of tensile stress produced by one layer is cancelled by the compressive stress in the next, ideally giving a net zero stress in the final coating. The values of compression and tension are determined using flat stress discs. Figure 12 shows the first capsule produced using a pressure cycling between 5 and 14 mTorr. In addition to cycling, the sputter gun power was reduced to 200 watts per gun from the previous 300 watts per Be gun based on preliminary results that showed that high deposition rates may be the primary cause of twisted structure formation.

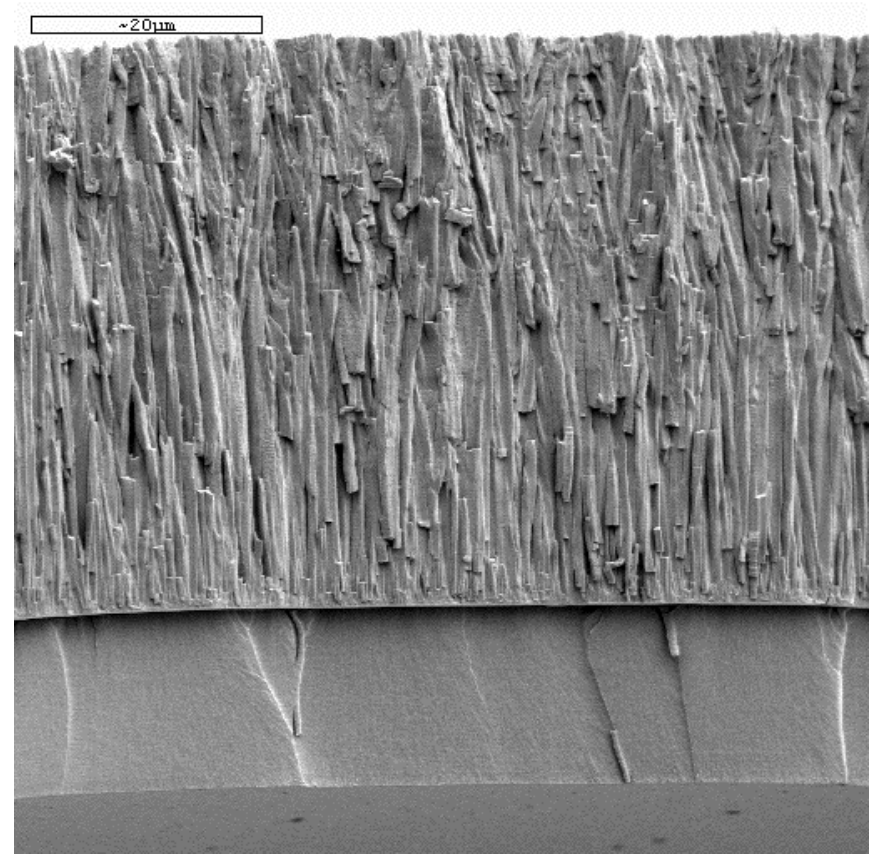

Figure 12. The argon sputter gas pressure was varied during the deposition of this capsule.

Pressure cycling has a tendency to produce additional debris as a result of the lower mean free path of the sputtered material at the higher Ar pressures. For this reason the cycling range was reduced to a 5 mTorr excursion around the balance point in later runs. In addition, runs with no pressure cycling was while holding the power level down at 200 watts per Be gun showed results similar to those with pressure cycling, with the twisted structure not forming and the columnar structure continuing through the thickness.

Since the targets can only provide about 70 microns of Be coating before requiring replacement we are currently attempting to make full thickness capsules using multiple runs. We are finding that in the range of 70 to 90 microns of thickness in most cases the growth morphology crosses over to a structure with the characteristics of PIG. The start of this alternative growth morphology does not typically coincide with the changing of the Be targets. It also does not coincide with changes in $\mathrm{Cu}$-doping concentration. In fact, to date we are unable to observe any effects of $\mathrm{Cu}$ doping on the microstructure. We currently are speculating that this alternative 
structure (i.e. PIG) is related to capsule-capsule abrasion, capsule-pan abrasion, or a combination of both. The basic idea is that either the debris generated by the abrasion is sticking to the capsules and nucleating this alternative growth or the type of surface damage created by the abrasion creates an alternative type of nucleation center on the capsule surface that lead to this growth.

\section{CONCLUSIONS}

By expanding the range of techniques used to metallurgically characterize the Be capsule materials produced by sputtering we have been able to identify a variety of interesting properties of thick Be materials formed on spherical mandrels including an unexpected non-c-axis texture to the growth. We have identified two barriers to the fabrication of full thickness columnar structure $\mathrm{Cu}$-doped $\mathrm{Be}$ capsules for NIF direct drive ICF targets. In the earliest runs, in which the deposition was stopped between daily runs, it was found that the material remained in the columnar structure however at sufficient thickness the growth morphology crossed from columnar to what appears to be particulate-initiated growth. In contrast to this, when deposition was finally able to run continuously another transition to a "twisted" growth structure, which appears to have a fairly low density, was observed to occur around 20 to 30 microns. By lowering the deposition rate we have been able to suppress the twisted structure and retain the columnar structure. However, with continuous deposition to thicknesses similar to the FTR capsules we again observe the cross-over to what appears to be particulate-initiated growth. We are currently exploring methods to suppress this cross-over, but this may require moving to a microstructure other than columnar.

The $\mathrm{Cu}$-doping of capsules appears to be successful to the accuracy to which it can be measured and we saw no evidence of effects of the $\mathrm{Cu}$-doping level on the microstructure. Accurate absolute density measurements remain elusive but an enhanced effort has been undertaken to correct this deficiency, as it is a critical material parameter.

\section{ACKNOWLEDGEMENTS}

We would like to thank Jeff Kass, Mark Wall, Rick Gross, and Jim Ferreira of LLNL for the timely processing and SEM imaging of the capsules from the first and second thick run as well as Ian Hutchinson and Rick Ryerson for electron probe measurements and analysis. We would also like to thank Pallas Papin for EPMA, optical and SEM metallography and FIB, Carl Necker for OIM, and Patricia Dickerson all of LANL for general assistance with metallographic analysis. Troy Barbee and Tom Beat of LLNL, David Cahill of UIUC, David Harding of UR, have provided important information and suggestions for sputter deposition, capsule fabrication, and characterization.

This work was performed under the auspices of the U.S. Department of Energy by University of California, Lawrence Livermore National Laboratory under contract W-7405-Eng-48. 


\section{REFERENCES (duplication will be fixed in Camera ready copy)}

${ }^{1} \mathrm{~S}$. W. Haan, et al., " Update on specifications for NIF ignition targets, and their rollup into an error budget ", Fusion Science and Technology, this issue

${ }^{2}$ R. B. Stephens, S. W. Haan, and D. C. Wilson, "Characterization Specifications for Baseline Indirect Drive NIF Targets," Fusion Science and Technology 41, 226 (2002).

${ }^{3}$ G. H. Miller, E. I. Moses, and C. R. Wuest, "The National Ignition Facility," Opt. Eng. 43, 2841 (2004).

${ }^{4}$ S. W. Haan, et al., " Update on specifications for NIF ignition targets, and their rollup into an error budget ", Fusion Science and Technology, this issue.

${ }^{5}$ B. W. McQuillan, A. Nikroo, D. A. Steinman, F. H. Elsner, D. G. Czechowicz, M. L. Hoppe, M. Sixtus, and W. J. Miller, "The P $\alpha$ MS/GDP Process for Production of ICF Target Mandrels," Fusion Technol. 31, 381 (1997).

${ }^{6}$ R. McEachern, C. Alford, R. Cook, D. Makowiecki, and R. Wallace, "Sputter Deposited Be Ablators for NIF Target Capsules,"Fusion Technol. 31, 435 (1997); R. C. Cook, M.

Anthamatten, J. P. Armstrong, S. A. Letts, R. L. McEachern, B. W. McQuillan, and M. Takagi "Recent Progress in the Development of Capsule Targets for the National Ignition Facility," Proc. of the SPIE 5228, 692 (2003).

${ }^{7}$ S. W. Haan, et al., " Update on specifications for NIF ignition targets, and their rollup into an error budget ", Fusion Science and Technology, this issue.

${ }^{8}$ P. B. MIRKARIMI, E. SPILLER, S. L. BAKER, V. SPERRY, D. G. STEARNS, E. M. GULLIKSON, "Developing a viable multilayer coating process for extreme ultraviolet lithography reticles”, J. Microlith., Microfab., Microsyst., 3, 139 (2004).

${ }^{9}$ Robert Field, LANL, private communication.

${ }^{10}$ Pallas Papin, LANL, private communication.

${ }^{11}$ Thomas Beat, LLNL, private communication. 